\title{
An immuno-electronmicroscopical study comparing vasopressin, oxytocin, substance $P$ and enkephalin containing nerve terminals in the nucleus of the solitary tract of the rat
}

\author{
P. VOORN and R. M. BUIJS* \\ Netherlands Institute for Brain Research IJdijk 28, 1095 KJ Amsterdam (The Netherlands)
}

(Accepted March 1st, 1983)

Key words: nucleus of the solitary tract — vasopressin — oxytocin — substance P — enkephalin — blood pressure

\begin{abstract}
Vasopressin, oxytocin, substance P and enkephalin fibers were demonstrated to terminate synaptically in the nucleus of the solitary tract. The detergent Triton X-100 proved to be indispensable for the demonstration of vasopressin and oxytocin while enkephalin and substance $\mathrm{P}$ could be visualized very well without it. The differences with respect to morphology between these 4 peptides disappeared when Triton X-100 was used in both groups.
\end{abstract}

Light microscopical immunocytochemical studies have revealed arginine-vasopressin (AVP) and oxytocin (OXT) to be present in an extensive fiber network throughout the brain $2,4,7$. In the limbic system by means of immuno-electronmicroscopy (IEM) the subcellular localization of AVP and, to a less extent, OXT was demonstrated ${ }^{3,6}$. A far more pronounced distribution of OXT compared with that of AVP, is found in the medulla oblongata: in the nucleus of the solitary tract (NTS), and the dorsal motor nucleus of the vagus 2,16 . In this area the immunocytochemical distribution of enkephalin (ENK) and substance $P$ (SP) $)^{5,18}$ displays a considerable overlap with that of AVP and OXT. Until now, it has not been shown whether these peptidergic fibers actually terminate in this region. In addition, relatively little is known about possible differences in ultrastructural localization of the peptides. Therefore the NTS was selected to compare the ultrastructural localization of OXT, AVP, ENK and SP in nerve endings and varicosities.

Male adult Wistar rats were perfusion-fixed with $2.5 \%$ glutaraldehyde, $1 \%$ paraformaldehyde in 0.1 $\mathrm{M}$ cacodylate buffer $\mathrm{pH}$ 7.3. The selected brain regions were excised and immersed in the same fixative for $2-3 \mathrm{~h}$ at $4{ }^{\circ} \mathrm{C}$. Fifty $\mu \mathrm{m}$ tissue sections were made on a vibratome. The immunocytochemical (PAP) procedure and the preparation of the tissue for electronmicroscopy were essentially the same as previously described ${ }^{3,4}$. Production and purification of the antisera has been described elsewhere $6,12,17$. The applied sera were rabbit AVP 125 and 126 (1:1000), absorbed with OXT-coupled Sepharose beads (1:400); rabbit OXT $0-1-\mathrm{V}$ antiserum $(1: 1000)$, absorbed with AVP-coupled beads (1:400). Rabbit Leu-Enk antiserum (1:800) and monoclonal rat SP antiserum (1:500) were kindly supplied by Dr. R. J. Miller and Dr. A. C. Cuello. To study the effects of Triton X-100, the detergent was used in a concentration of 0.2 or $0.4 \%$ until the $\mathrm{DAB}$ incubation, or $0.5 \%$ in the first antiserum and the following incubations in $0.2 \%$.

For the demonstration of AVP/OXT immunoreactivity, the use of Triton in all incubation steps proved to be a necessity. The conditions for optimal staining, viz. staining intensity of individual fibers versus the number of stained fibers, in combination with the least ultrastructural damage were found using a Triton concentration of $0.5 \%$ in the first antiserum and $0.2 \%$ in the remaining incubations. ENK- and SP-immunoreactive fibers were easily visualized without using Triton at all. AVP/OXT immunoreactivity was found in unmyelinated axons and varicosities. In ax-

* To whom correspondence should be addressed. 
ons, reaction product was localized in large granular vesicles (LGV) (70-100 nm) or diffusely in the cytoplasm. In terminals and varicosities, the reaction product was associated with most organelles: in LGV (2-4 mostly in the periphery of the profile; $70-100$ $\mathrm{nm})$ and plasmalemma, around small synaptic vesicles with a clear vesicle-like appearance $(30-50 \mathrm{~nm})$, around microtubuli and mitochondria, and diffusely in the cytoplasm (Fig. 1). Using purified antisera (with higher Triton concentrations) generally more LGV were found than with the unpurified sera (Fig. 1c,d). Also more 'free deposits' of reaction product in the cytoplasm and less well-preserved clear vesicles seemed to be present. Out of 93 OXT- and 114 AVPcontaining profiles, respectively, 13 and $15 \%$ exhibited a synaptic specialization. Apart from one vasopressinergic axo-somatic contact, only axo-dendritic junctions were observed (Fig. 1). No synaptic input could be seen on the AVP/OXT fibers. No distinction could be made between AVP or OXT, neither in synaptic morphology nor in their post-synaptic elements.

ENK- and SP-immunoreactivity was found in unmyelinated axons and varicosities. The subcellular
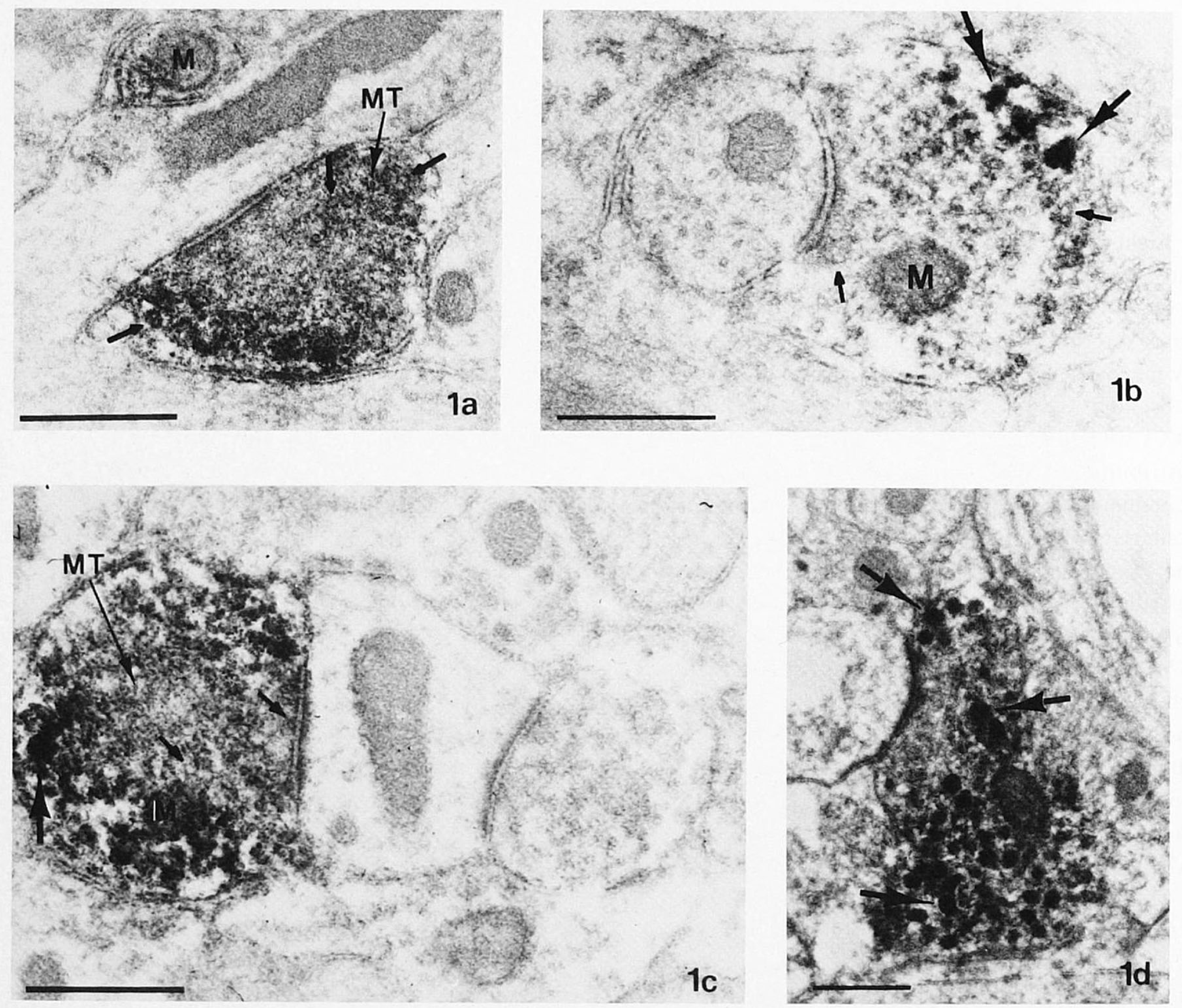

Fig. 1. Vasopressin- (a,b) and oxytocin- $(\mathrm{c}, \mathrm{d})$ containing terminals forming axodendritic synaptic junctions in the nucleus of the solitary tract. Triton X-100 detergent was used during all antiserum incubations. Note the presence of $70-100 \mathrm{~nm}$ large granular vesicles (large arrows) and the concentration of clear vesicles (CV) (small arrows) above the synaptic junctions. Figs. c,d were obtained after using, respectively, AVP-adsorbed anti-oxytocin or OXT adsorbed anti-vasopressin serum, MT, microtubuli-like structures; M, mitochondrion. $\mathrm{Bar}=0.5 \mu \mathrm{m}$. 

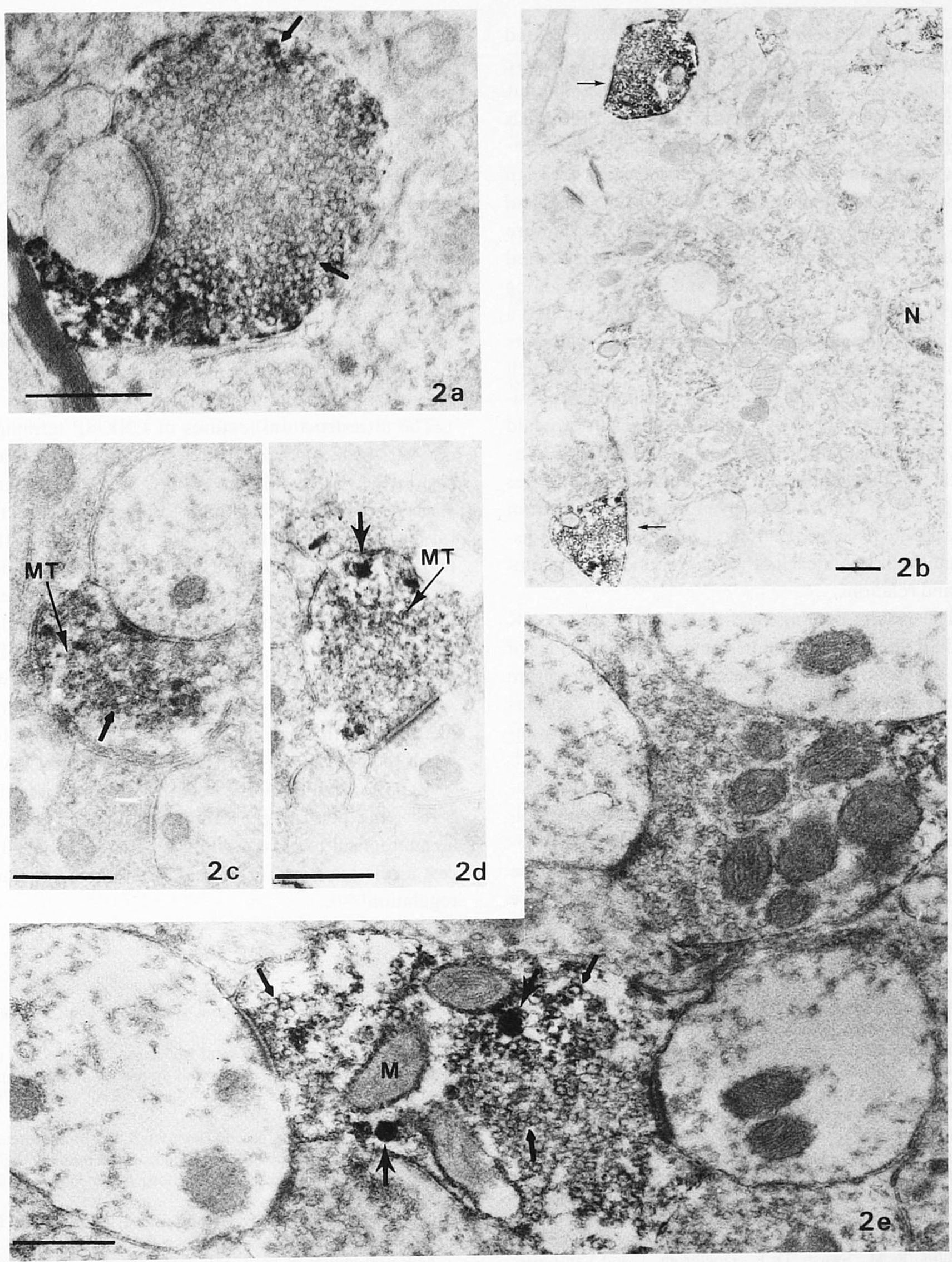

Fig. 2. Substance $P(a-c)$ and enkephalin (d) containing terminals forming synaptic contacts with dendritic spine (a) cell soma (b) or dendrites $(\mathrm{c}-\mathrm{e})$. Note the difference in ultrastructural preservation of the membranes and in the size of the clear vesicles between the Triton X-100-treated material (c-d) and the non-detergent treated material. Size of clear vesicles in a and e 40-60 nm. Abbreviations as in Fig. $1 ; \mathrm{N}$, nucleus. Bar $=0.5 \mu \mathrm{m}$. 
localization of reaction product in Triton-treated material was identical to that in the AVP/OXT nerve profiles (see above; cf. Figs. 1 and 2 c,d). Without using Triton, however, the varicosities and terminals were densely packed with clear vesicles. These vesicles seemed to have a larger electronlucent core than those in the Triton-treated sections (40-60 nm and 30-50 nm respectively, Fig. $2 \mathrm{a}, \mathrm{b}$ and e). LGV were found less frequently than with Triton-treated material. Out of 152 ENK- and 94 SP-containing nerve endings, respectively, 62 and $57 \%$ exhibited a synaptic specialization. The post-synaptic elements were dendritic spines or shafts and occasionally cell somata (Fig. 2b). The labeled terminals and varicosities did not establish axo-axonal contacts, nor could any synaptic input be observed. Often ENK/SP synaptic profiles contacted two unlabeled dendrites (Fig. 2e), or dendrites received manifold input from one ENK/SP axon. ENK and SP fibers were indistinguishable from each other in synaptical morphology and relations.

The results demonstrate that AVP, OXT, ENK and SP fibers form synaptic contacts in the nucleus of the solitary tract. A comparison of the ultrastructure shows that the non-Triton-treated material contains more 40-60 $\mathrm{nm}$ clear vesicles, while the Tritontreated elements show more LGV, clear vesicles with smaller electronlucent core and more pronounced labeling around microtubuli. These phenomena indicate that Triton X-100 largely destroys the clear vesicle membrane, while interfering much less with the microtubuli (cf. ref. 12). It is clear that extreme care should be taken in interpreting the subcellular locali-

1 Bohus, B., Versteeg, C. A. M. and De Jong, W., Implication of a brain peptidergic system in the pathogenesis of neurogenic hypertension, P. J. Janetta, M. Dujovny and R. Segal (Eds.), Raven Press, NY, 1982.

2 Buijs, R. M., Intra- and extrahypothalamic vasopressin and oxytocin pathways in the rat; pathways to the limbic systems, medulla oblongata and spinal cord, Cell Tiss. Res., 192 (1978) 423-435.

3 Buijs, R. M. and Swaab, D. F., Immuno-electronmicroscopical demonstration of vasopressin and oxytocin synapses in the limbic system of the rat, Cell Tiss. Res., 204 (1979) $355-365$.

4 Buijs, R. M., Swaab, D. F., Dogterom, J. and Van Leeuwen, F. W., Intra- and extrahypothalamic vasopressin and oxytocin pathways in the rat, Cell Tiss. Res., 186 (1978) $423-433$.

5 Cuello, A. C. and Kanazawa, I., The distribution of sub- zation of peroxidase reaction product with respect to the actual peptide localization. It is through the indispensability of the detergent Triton X-100 in demonstrating AVP/OXT immunoreactivity in the NTS and the ready staining of ENK/SP profiles without it that the labeling procedures and the ultrastructural features of the ENK/SP labeled structures stand out against those containing AVP/OXT. A possibly higher peptide concentration in ENK/SP fibers compared to that in AVP/OXT fibers, different membrane properties regarding penetration of antisera, or a different way of incorporation of AVP and OXT in precursor molecules rendering them less accessible to antisera may account for this phenomenon.

The ultrastructural features of ENK/SP terminals and varicosities match those described in other brain regions $29,10,14,15$, while the AVP/OXT terminals and varicosities in the NTS exhibit the same ultrastructural features as those in the limbic system ${ }^{3}$. Since the two extrahypothalamic AVP/OXT fiber systems differ from the hypothalamo-neurohypophyseal system in the size of the hormone-containing large granular vesicles (80-100 nm and 140-160 nm, respectively) (ref. 3 and this study). It is likely that there are two AVP/OXT containing systems, one projecting into the brain, with small, $80 \mathrm{~nm} \mathrm{LGV}$, and another towards the neural lobe, with large, 140-160 nm LGV.

As part of the baroreflex control is centered in the $\mathrm{NTS}^{13}$, the peptidergic terminations might provide an anatomical basis to account for the data that suggest a role for AVP, OXT and SP in blood pressure regulation $1,8,11$.

stance $\mathrm{P}$ in the central nervous system, J. comp. Neurol., 178 (1978) 129-156.

6 Cuello, A. C., Galfre, G. and Millstein, C., Detection of substance $\mathrm{P}$ in the central nervous system by a monoclonal antibody, Proc. nat. Acad. Sci. U.S.A., 76 (1979) 3532-3536.

7 DiFiglia, M., Aronin, N. and Leeman, S. E., Immunoreactive substance $P$ in the substantia nigra of the monkey: light and electron microscopic localization, Brain Research, 23 (1982) 381-388.

8 Gillis, R. A., Helke, C. J., Hamilton, B. L., Norman, W. P. and Jacobowitz, D. M., Evidence that substance $\mathrm{P}$ is a neurotransmitter of baro- and chemoreceptor afferents in nucleus tractus solitarius, Brain Research, 181 (1980) 476-481.

9 Helenius, A. and Simons, K., Solubilization of membranes by detergents, Biochem. biophys. Acta, 415 (1975) 29-79. 
10 Hunt, S. P., Kelly, J. S. and Emson, P. C., The electron microscopic localization of methionine-enkephalin within the superficial layers (I and II) of the spinal cord, Neuroscience, 5 (1980) 1871-1890.

11 Matsuguchi, H., Sharabi, F. M., Gordon, F. J., Johnsson, A. K. and Schmid, P. G., Blood pressure and heart rate responses to microinjection of vasopressin into the nucleus tractus solitarius region of the rat, Neuropharmacology, 21 (1982) 687-693.

12 Miller, R. J., Chang, K.-J., Cooper, B. and Cuatrecasas, P., Radioimmunoassay and characterization of enkephalin in rat tissues, J. biol. Chem., 253 (1978) 531-538.

13 Palkovits, H. and Zaborsky, L., Neuroanatomy of central cardiovascular control. Nucleus tractus solitarius: afferent and efferent neuronal connections in relation to the baroreceptor reflex arc. In W. de Jong, A. P. Provoost and A. P. Shapiro (Eds.), Hypertension and Brain Mechanisms. Progress in Brain Research, Vol. 47, Elsevier/North Holland, Amsterdam, 1977, pp. 9-34.

14 Pickel, V. M., Joh. T. H., Reis, D. J., Leeman, S. E., and Miller, R. J., Electromicroscopic localization of substance
$\mathrm{P}$ and enkephalin in axon terminals to dendrites of catecholaminergic neurons, Brain Research, 160 (1979) 387-400.

15 Priestley, J. V., Ultrastructural localization of substance P and enkephalin in the substantia gelatinosa of the spinal trigeminal nucleus, Brit. J. Pharmacol., 74 (1981) 893-894.

16 Sawchenko, P. E. and Swanson, L. W., Immunohistochemical identification of neurons in the paraventricular nucleus of the hypothalamus that project to the medulla or to the spinal cord in the rat, J. comp. Neurol., 205 (1982) 260-272.

17 Swaab, D. F. and Pool, C. W., Specificity of oxytocin and vasopressin immunofluorescence, J. Endocrinol., 66 (1975) 263-272.

18 Uhl, G. R., Goodman, R. R., Khar, M. J., Childers, S. R. and Snyder, S. H., Immunohistochemical mapping of enkephalin-containing cell bodies, fibers and nerve terminals in the brainstem of the rat, Brain Research, 166 (1979) 75-94.

19 Van Leeuwen, F. W. and Swaab, D. F., Specific immunoelectronmicroscopic localization of vasopressin and oxytocin in the neurohypophysis of the rat, Cell Tiss. Res., 177 (1977) 493-501. 